\title{
Exploitation of the interaction of measles virus fusogenic envelope proteins with the surface receptor CD46 on human cells for microcell-mediated chromosome transfer
}

\author{
Motonobu Katoh1,2, Yasuhiro Kazuki2,3,5, Kanako Kazuki³, Naoyo Kajitani33, Masato Takiguchi³, Yuji Nakayama4, \\ Takafumi Nakamura6,7 and Mitsuo Oshimura*2,3,5
}

\begin{abstract}
Background: Microcell-mediated chromosome transfer (MMCT) is a technique by which a chromosome(s) is moved from donor to recipient cells by microcell fusion. Polyethylene glycol (PEG) has conventionally been used as a fusogen, and has been very successful in various genetic studies. However, PEG is not applicable for all types of recipient cells, because of its cell type-dependent toxicity. The cytotoxicity of PEG limits the yield of microcell hybrids to low level (10${ }^{6}$ to $10^{-5}$ per recipient cells). To harness the full potential of $\mathrm{MMCT}$, a less toxic and more efficient fusion protocol that can be easily manipulated needs to be developed.

Results: Microcell donor $\mathrm{CHO}$ cells carrying a human artificial chromosome (HAC) were transfected with genes encoding hemagglutinin (H) and fusion (F) proteins of an attenuated Measles Virus (MV) Edmonston strain. Mixed culture of the $\mathrm{CHO}$ transfectants and MV infection-competent human fibrosarcoma cells (HT1080) formed multinucleated syncytia, suggesting the functional expression of the MV-H/F in the $\mathrm{CHO}$ cells. Microcells were prepared and applied to HT1080 cells, human immortalized mesenchymal stem cells (hiMSC), and primary fibroblasts. Drug-resistant cells appeared after selection in culture with Blasticidin targeted against the tagged selection marker gene on the HAC. The fusion efficiency was determined by counting the total number of stable clones obtained in each experiment. Retention of the HAC in the microcell hybrids was confirmed by FISH analyses. The three recipient cell lines displayed distinct fusion efficiencies that depended on the cell-surface expression level of CD46, which acts as a receptor for MV. In HT1080 and hiMSC, the maximum efficiency observed was 50 and 100 times greater than that using conventional PEG fusion, respectively. However, the low efficiency of PEG-induced fusion with HFL1 was not improved by the MV fusogen.
\end{abstract}

Conclusions: Ectopic expression of MV envelope proteins provides an efficient recipient cell-oriented MMCT protocol, facilitating extensive applications for studies of gene function and genetic corrections.

\section{Background}

Microcell fusion is a method which enables the transfer of a single mammalian chromosome or its fragment from donor to recipient cells. This method consists of five essential steps: 1) micronucleation of donor cells; 2) enucleation of the micronucleated cells; 3 ) isolation of micro-

* Correspondence: oshimura@med.tottori-u.ac.jp

${ }^{2}$ Chromosome Engineering Research Center, Tottori University, Yonago 6838503, Japan

Full list of author information is available at the end of the article cells; 4) fusion of microcells with recipient cells; and 5) selection of viable microcell hybrid clones. Microcellmediated chromosome transfer (MMCT) offers several advantages for the transfer of genetic material between mammalian cells. Thus, megabase-sized stretches of an intact chromosome can be moved, which tend to be stable and freely segregating in recipient cells [1]. MMCT to patient-derived cells, followed by functional complementation assays, has been used for the genetic mapping and identification of genes responsible for hereditary reces- 
sive disorders and for tumor suppressor genes [2-5]. Other fields have taken advantage of MMCT for addressing genomic instability, genomic imprinting, chromatin modification, and structural and spatial organization of the genome [1,6-9]. Transfer of human chromosome fragments or artificially engineered chromosomes into embryonic stem cells has also successfully produced transchromosomic (Tc) animals [10,11]. These Tc animals have been used as sources of therapeutics and as models of human disorders such as Down's syndrome $[2,12,13]$. MMCT has also been applied to construction and manipulation of artificial chromosome vectors for potential human gene therapies $[14,15]$. MMCT has thus paved the way for the use of mammalian chromosomes as gene delivery vectors.

The most commonly used reagent for microcell fusion is high molecular weight polyethylene glycol (PEG) [16]. Since the establishment of standard method in the 1980s, the MMCT has been achieved with a specific class of recipient cells, for which the introduction of considerable changes in the fusion protocol was not necessary. However, little is known about the fusion mechanism of PEG. PEG may cause the redistribution of intramembrane molecules within the plasma membrane. This ability of PEG has been attributed to the ordering of water induced by a high concentration of polymer [17]. As well as inducing cell fusion, the PEG treatment concomitantly results in extensive cell damage and loss of cell viability because of the induced cytotoxicity [18]. Sensitivity to the PEG cytotoxicity is known to be cell type-dependent and is regulated by the lipid composition of the cell membrane $[19,20]$. Consistent with these data, the success of microcell fusion by PEG seems to depend on the particular combination of donor and recipient cells. Characterization and engineering of the lipid composition of cell membranes in order to avoid the PEG cytotoxicity is an intriguing but daunting task [21]. Therefore, to exploit novel applications of the MMCT, a more efficient, less toxic and more easily manipulated fusion protocol than PEG treatment needs to be developed.

One well-documented naturally occurring membrane fusion event is that which occurs during the infection of host cells by enveloped viruses. The measles virus (MV), which causes the acute contagious disease of measles, has an envelope protein complex that is used for both virus attachment and membrane fusion [22]. The complex is composed of two integral membrane proteins, the hemagglutinin $(\mathrm{H})$ and fusion $(\mathrm{F})$ proteins. $\mathrm{MV}-\mathrm{H}$ is the transmembrane glycoprotein responsible for the interaction of the virus with its cellular receptors. Two receptors have been identified for the live attenuated Edmonston B vaccine strain of MV (MV-Edm): the ubiquitously expressed regulator of complement activation CD46 and the lymphocyte-specific protein of the immunoglobulin
(Ig) superfamily, SLAM. The H protein mediates attachment to either one of these cell surface receptors, and signals to the $\mathrm{F}$ protein to trigger membrane fusion. Infected MV propagates both by virus release and reinfection, and by cell-cell fusion. Virus-induced cell-cell fusion plays an important role in the propagation and pathogenicity of MV. MV-Edm has been shown to be selectively oncolytic [23]. This effect is due to the fact that CD46 is upregulated in tumor cells, thereby allowing MV-Edm to selectively target and destroy tumor cells through this receptor [24]. Furthermore, MV-Edm has been retargeted by engineering the $\mathrm{H}$ protein to efficiently enter tumor cells via alternative cellular receptors $[25,26]$. These previous studies prompted us to test the idea that transduction of genes encoding the $\mathrm{H}$ and $\mathrm{F}$ proteins into non-human donor cells may lead to the production of fusogenic microcells (Figure 1).

In this study, we tested the feasibility of using the MV viral fusion machinery as an alternative to PEG for microcell fusion. An expression vector encoding the MV-H and $\mathrm{F}$ genes was transfected into a $\mathrm{CHO}$ cell line which carries an artificial human chromosome (HAC) tagged with a drug-resistant selection marker. Functional expression of the transfected MV-H/F plasmids was confirmed by syncytium formation in a mixed culture of the engineered CHO cells with CD46-expressing HT1080 (fibrosarcoma) cells. Microcells were prepared from the donor cells and overlaid on HT1080, hiMSC (human immortalized mesenchymal stem cells), or HFL-1 (primary fibroblasts, derived from fetal lung) recipient cells. Drug-resistant colonies were obtained by selection culture and introduction of the HAC was confirmed by FISH analysis. The efficiency of microcell fusion with HT1080 or hiMSC using the MV fusogen was higher than that using PEG by an order of magnitude whereas the low efficiency of PEGinduced fusion with HFL1 was not improved by the MV fusogen. Difference in the fusion efficiency by the MV fusogen may be explained by the expression level of the CD46 receptor on these recipient cells. Potential extension of the host tropism of fusogenic microcells is also discussed.

\section{Results}

Introduction of the MV-F/H genes confers human celldirected fusion ability on $\mathrm{CHO}$ cells

As the first step towards microcell fusion using an MV fusogen, we transfected expression cassettes encoding the $\mathrm{MV}-\mathrm{H}$ and $-\mathrm{F}$ proteins, and the Neo/DsRed plasmid, into $\mathrm{CHO}(\mathrm{HAC})$ cells, which carry a HAC engineered from human chromosome 21 [14]. In the course of construction, the HAC was tagged with a Blasticidin-resistant (Bsr) gene and GFP. After transfection, the cells were cultured in medium containing the antibiotic G418. The G418-resistant cell population was recovered and desig- 


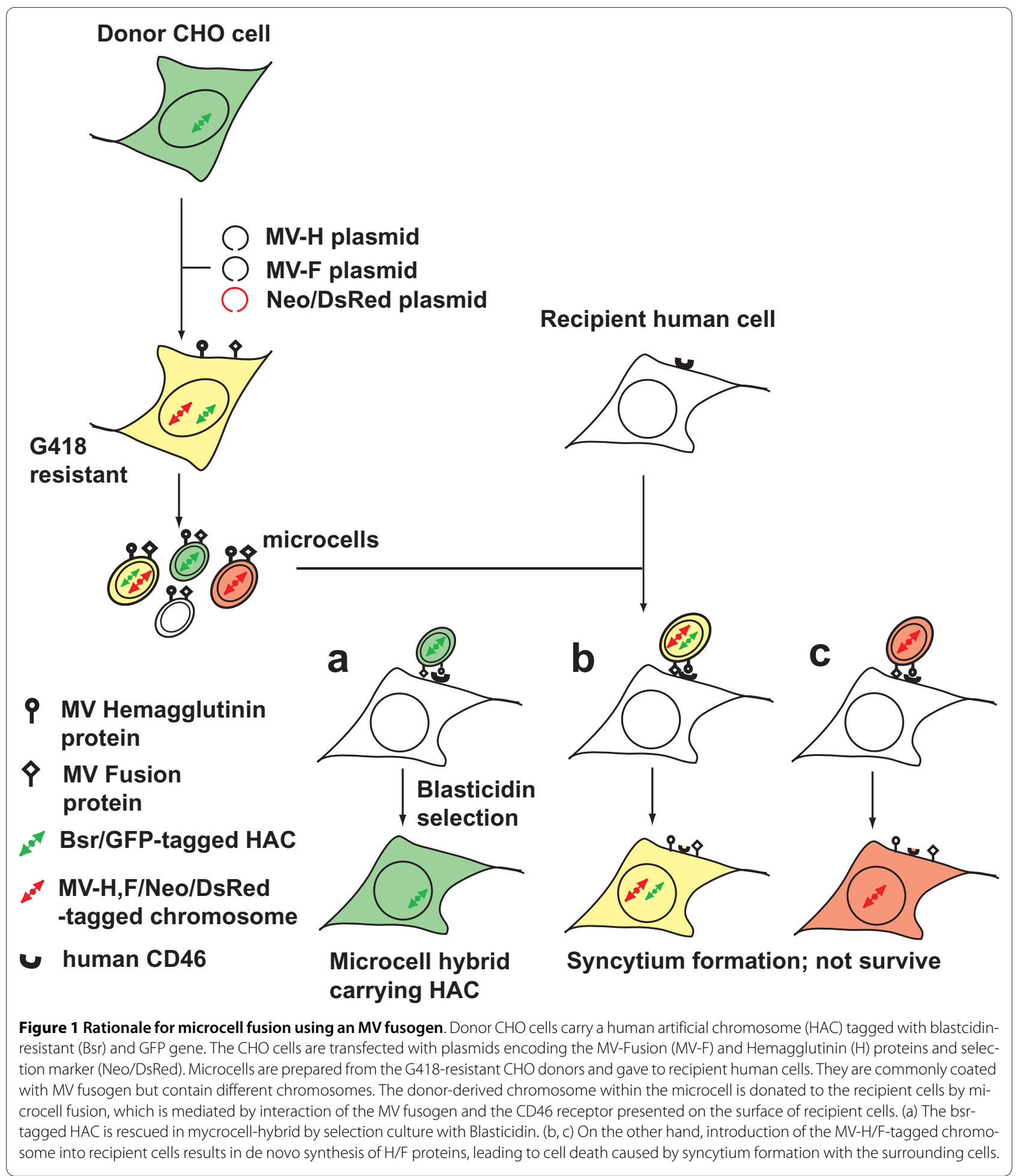

nated as CHO4H6.1M. Most of them expressed both GFP and DsRed fluorescences (Figure 2a, b, c). It is known that transfection of MV-H/F into human cells induce syncytium formation by homofusion (Figure 2e). Unlike the case with MV-H/F transfected human cells, no syncytium was observed during propagation of the $\mathrm{CHO} 4 \mathrm{H} 6.1 \mathrm{M}$ cells (Figure 2a). The absence of syncytium formation was consistent with the prediction that the fusion machinery of the human specific-ecotropic MV does not function in rodent cells. The surface expression of the $\mathrm{H}$ protein was assessed by flowcytometry analysis. About one third of the cells showed slight expression of the $\mathrm{H}$ protein (addi- 

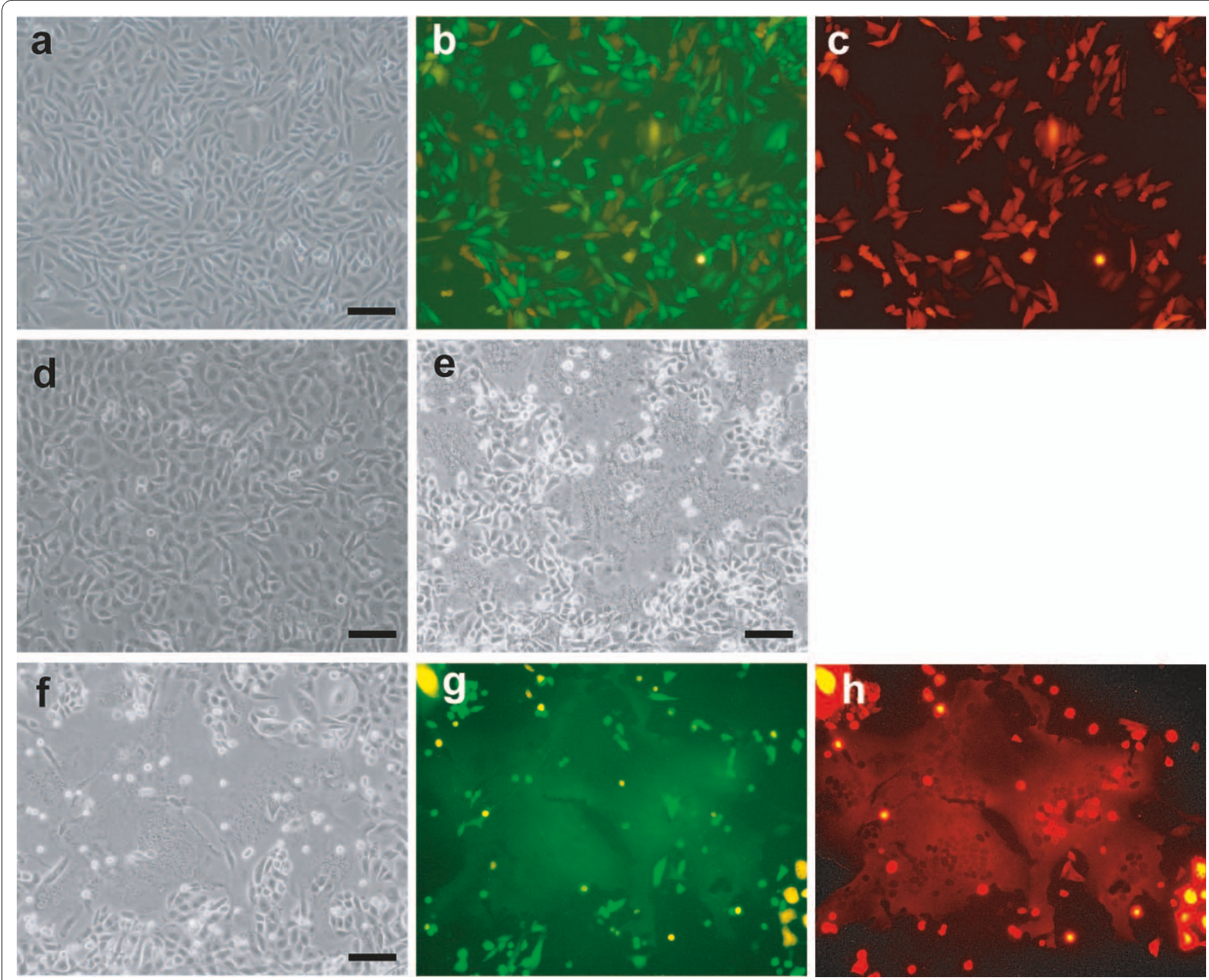

Figure 2 Genetically engineered CHO cells can fuse with human cells. G418-resistant CHO cells (a) expressed the GFP (b) and/or the fluorescent marker DsRed (c). An equal number of the CHO cells (a) and HT1080 cells (d) were mixed, and photographed 3 days later (f, g, h). Similar to the MV-F/ $\mathrm{H}$-transfected HT1080 cells (e), large multinucleated syncytia were observed (f). Syncytia made by heterofusion of CHO and HT1080 expressed GFP and DsRed fluorescence $(\mathrm{g}, \mathrm{h})$. The panels $\mathrm{a}, \mathrm{b}, \mathrm{c}$ and $\mathrm{f}, \mathrm{g}$, h show the same cell field, respectively. Scale bar, $10 \mu \mathrm{m}$. Photographs were taken under phase contrast $(a, d, e, f)$ or fluorescence $(b, c, g, h)$ microscopic conditions.

tional file 1). It is generally expected that co-transfection of linearlized plasmids are concatenated and integrated together in the same chromosomal locus. Although the concomitant expression of $\mathrm{H}$ and $\mathrm{F}$ was not directly tested, a part of $\mathrm{H}$ protein expressing cells may also express $\mathrm{F}$ protein.

We then tested the functional expression of $\mathrm{H}$ and $\mathrm{F}$ on the cell surface. The $\mathrm{CHO} 4 \mathrm{H} 6.1 \mathrm{M}$ cells were co-cultured with the human tumor cell line HT1080. After culture for $24 \mathrm{~h}$, a multinucleated syncytium was observed and further expanded (Figure 2f, g, h). Syncytia contained fluorescence-positive and -negative nuclei that were supposed to be derived from CHO and HT1080 cells, respectively (Figure $2 \mathrm{~h}$ ). The occurrence of heterofusion, and the absence of homofusion, suggested that although the transfected $\mathrm{MV}-\mathrm{H} / \mathrm{F}$ proteins did not induce fusion in the $\mathrm{CHO}$ cells, they were functionally expressed on the $\mathrm{CHO}$ cell surface and could mediate fusion with human cells.

\section{The genetically engineered $\mathrm{CHO}$ cells produce fusogenic microcells}

The ability of the CHO4H6.1M cells to function as donor cells for microcell fusion was then tested. Microcells were prepared from donor cells $\left(\sim 8 \times 10^{6}\right)$ that expressed DsRed and/or GFP (Figure 2a, b, c). These fluorescent microcells $\left(\sim 8 \times 10^{5}\right)$ were overlaid on $2 \times 10^{6}$ of HT1080 cells. Following $24 \mathrm{~h}$ incubation, weak fluorescence was detected in a few recipient cells (Figure 3a), while most of fluorescent microcells were retained as distinct particles. Fluorescence in recipient cells was explained by either 


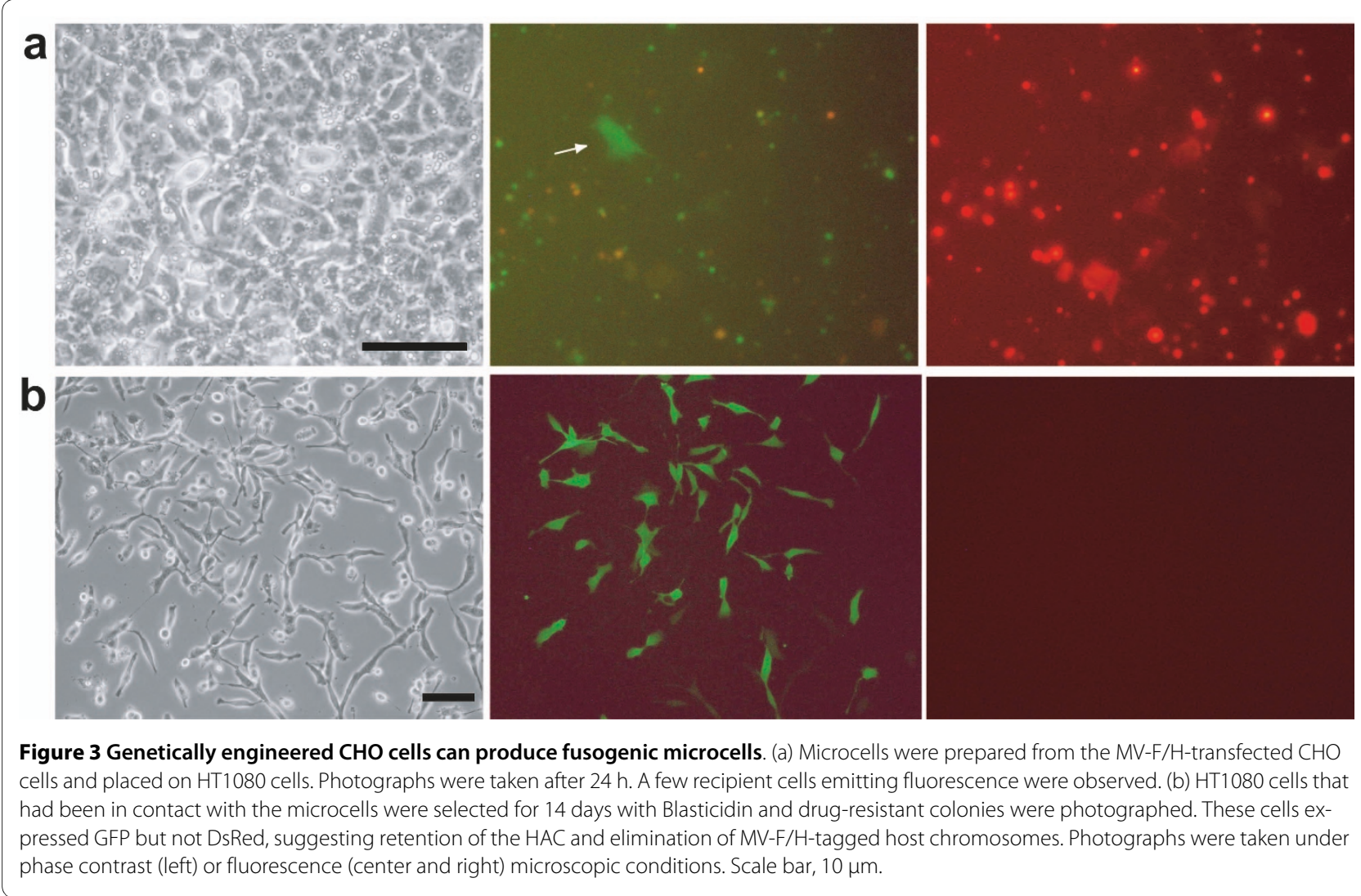

transmission of the fluorescent protein from the microcells or de novo synthesis in microcell hybrids. To confirm that the donor chromosome was transferred, the cultures were expanded and treated with Blasticidin which selects for cells carrying tagged HAC. A total of 80 drug-resistant colonies were detected following Blasticidin selection. Selected colonies were picked up and propagated for the following analysis. Unlike the case with $\mathrm{MV}-\mathrm{H} / \mathrm{F}$ transfected human cells, no syncytium was observed during propagation of the Blasticidin-resistant clones.

FISH analysis was performed with the GFP expressing cells for the detection of the HAC transfer (Figure 4). Alphoid satellite probe derived from human chromosome 21 hybridized with endogenous chromsomes 21 and 13, due to their sequence similarity [14]. In addition to these endogenous chromosomes, the probe detected a minichromosome, which is not present in parental HT1080 cells (data not shown). Since the HAC was constructed by deleting almost all sequences from long and short arm of the chromosome 21, it retains alphoid satellite as substantial contents [14]. The estimated size of the HAC is less than $10 \mathrm{Mb}$, which corresponds to $1 / 5$ of the original human chromosome 21. In comparison to the endogenous chromosome 21, the size of the minichromosome matched to the prediction. Obvious change in the karyotype of the host cells was not detected. These results demonstrated the introduction of the HAC by microcell fusion without disturbance of the host chromosomes.

It was noted that the colonies expressed GFP but not DsRed (Figure $3 \mathrm{~b}$ ). A possible explanation as to why the DsRed-expressing cells carrying HAC were not detected is that a microcell hybrid which received the donor chromosome tagged by DsRed/neo together with MV-H/F genes could not survive because of homofusion or fusion between surrounding cells. To test the elimination of the neo gene associated with the DsRed gene, Blasticidinresistant clones were assessed for the sensitivity to G418 (additional file 2). All the clones tested were sensitive to G418. These results indicated that stable microcell hybrids were obtained by excluding the DsRed/neotagged donor chromosomes. These data proved that chromosome transfer was successfully achieved by microcell fusion via an MV fusogen.

\section{The MV fusogen is more efficient than PEG for microcell hybrid production}

We next compared the efficiency of microcell fusion by MV fusogen with that by PEG. For this purpose, different amount of microcells were applied to the fixed number of recipient HT1080 cells. From $5 \times 10^{7}$ of CHO4H6.1M cells, $\sim 5 \times 10^{6}$ of microcells were obtained. These micro- 


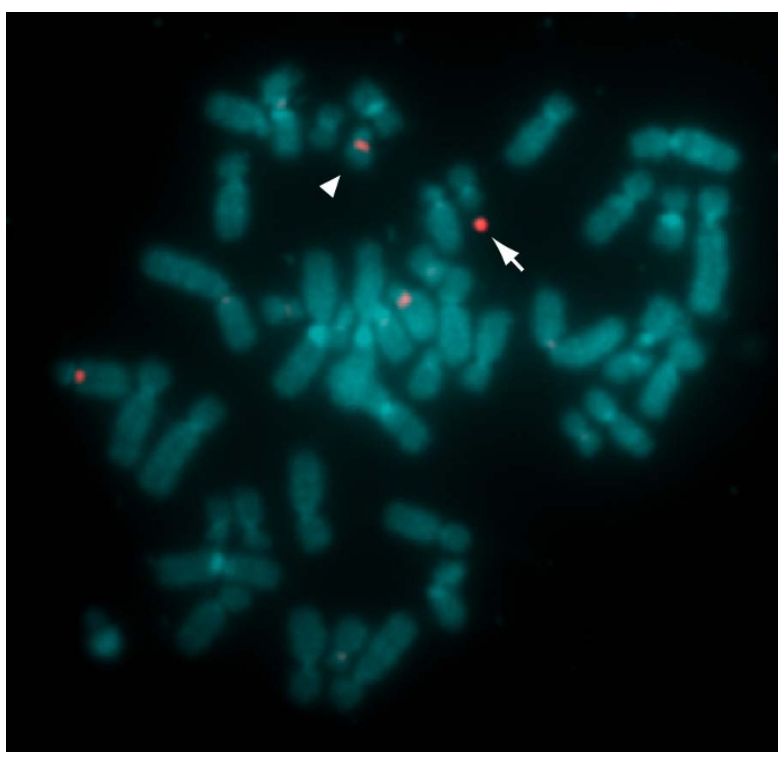

Figure 4 Detection of chromosome transfer from the microcell to the donor cell. Recipient cells exposed to the microcells, and selected by Blasticidin treatment, were analyzed by fluorescence in situ hybridization to detect cells containing the transferred HAC. The HAC is delineated using an alphoid satellite probe derived from human chromosome 21, and is shown in red (arrow). The alphoid satellite probe detects endogenous chromosomes 13 and 21 (arrowhead), in addition to the HAC, due to their sequence similarity.

cells were aliquoted in five fractions with the ratio of $1,2,4,6,11$, and applied to a fixed number $\left(2 \times 10^{6}\right)$ of recipient cells. As a control, the conventional fusion experiment using PEG was performed with the parental HAC donor cell line CHOkkpqG4. Microcell fusion was determined as the number of drug-resistant colonies following selection culture with Blasticidin (Figure 5). The fusion efficiency by MV fusogen was consistently, and at most 10 times, higher than that by PEG (Table 1). Whereas fusion induced by PEG was gradually increased in a microcell dose-dependent manner, fusion induced by

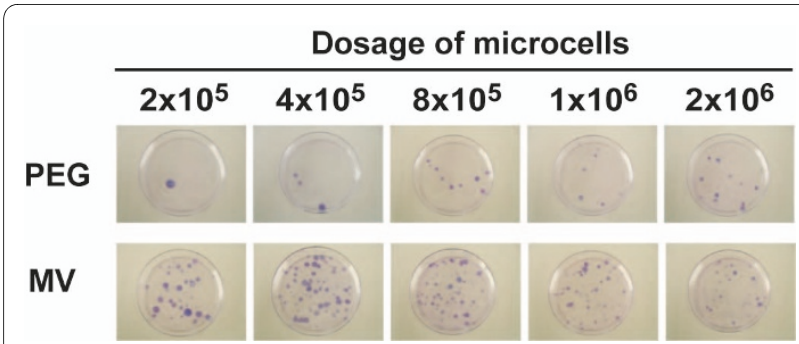

Figure $\mathbf{5}$ The MV fusogen is more efficient than PEG for microcell hybrid production. Microcells collected from 24 flasks $\left(\sim 5 \times 10^{6}\right)$ were fractionated into 5 dosing amounts $\left(2 \times 10^{5}, 4 \times 10^{5}, 8 \times 10^{5}, 1 \times 10^{6}, 2\right.$ $\left.\times 10^{6}\right)$ and added to (MV), or fused with (PEG), $2 \times 10^{6}$ of HT1080 cells in a 60-mm dish. On the following day, fused cells were plated onto two 100-mm dishes. Following selection culture for 14 days, drug-resistant colonies were stained with Giemsa and photographed. the MV fusogen did not show dose dependency. Input over threshold amount of microcells with MV fusogen seemed to reduce the appearance of microcell hybrids.

\section{Efficiency of microcell fusion via the MV fusogen differs with different recipient cells}

We next determined whether the relatively high microcell fusion efficiency observed using the MV fusogen and HT1080 cells at optimal conditions could also be observed with other cell types. We therefore compared the fusion efficiency of the MV-fusion system using HT1080 with that using a mesenchymal stem cell line hiMSC and primary fibroblast cells HFL-1. A fixed number of microcells was applied to different numbers of recipient cells. After selection culture for 2 weeks, the number of drug-resistant colonies was counted (Figure 6a). Irrespective of the number of recipient cells, MVfusion consistently induced more resistant colonies than PEG fusion in the HT1080 cell cultures. Although the colony number obtained by MV-fusion in hiMSC cells was approximately half of that in HT1080 cells, the hiMSC were also responsive to MV fusion. In contrast, difference in an order of magnitude was not observed between MV- and PEG-induced resistant colonies using the HFL-1 cells. The PEG-induced fusion efficiency using HFL-1 cells was quite low compared to the other cell lines and this low level of fusion was not overcome using the MV fusogen.

In HT1080 and hiMSC, it was noted that MV fusion induced resistant colonies in small numbers of recipient cells in which no colonies were induced by PEG fusion. Fusion efficiency defined as frequency of drug resistantcolony formation per recipient cells scored highest $\left(\sim 10^{-}\right.$ $4)$, when small number of recipient cells $\left(2 \times 10^{4}\right)$ were used. In HT1080 and hiMSC, the maximum efficiency was 50 and 100 times greater than that using conventional PEG fusion, respectively.

For drug-resistant cells, FISH analysis was performed for the detection of transferred HACs (Figure 7). In addition to the endogenous chromosomes 21 and 13, the alphoid satellite probe detected a minichromosome, which is not present in parental HT1080, hiMSC, and HFL-1 cells. In comparison to the endogenous chromosome 21 , the size of the minichromosomes matched to the prediction. In HFL-1, the alphoid probe detected a pair of endogenous chromosomes 21 and 13, whereas in HT1080 and hiMSC, the probe detected a single chromosome 21. While HFL-1 is primary cells, HT1080 and hiMSC were immortalized by the transformation [27]. Aberrant chromosome number in HT1080 and hiMSC may be explained by the transformation. The result obtained by the PEG fusion (data not shown) was consistent with that of the MV fusion. These results demonstrated that the HAC was transferred to the recipient cells 
Table 1: Yield of drug-resistant microcell hybrids by using MV fusogen and PEG

\begin{tabular}{clcc}
\hline Amount of applied microcells & Colony number 1 & & \\
\cline { 2 - 4 } & & MV & PEG \\
\hline $2 \times 10^{5}$ & 51 & 5 \\
$4 \times 10^{5}$ & 86 & 6 \\
$8 \times 10^{5}$ & 94 & 15 \\
$1 \times 10^{6}$ & 75 & 13 \\
$2 \times 10^{6}$ & 60 & 22 \\
\hline
\end{tabular}

1. Number of Blasticidin-resistant colonies obtained from $2 \times 10^{6}$ of HT1080 cells.

by microcell fusion without disturbance of the host chromosomes.

\section{Efficiency of microcell fusion may depend on the surface density of the CD46 receptor}

$\mathrm{MV}$ is known to be a potent and specific oncolytic agent that causes cytopathic effects due to extensive syncytium formation. A previous study reported that the extent of intercellular fusion in MV-infected human cells including HT1080, was determined by the surface density of CD46 [28]. These data suggested that the extent of microcell fusion may also be affected by the surface density of CD46. Therefore, we measured the surface density of CD46 in the three cell lines using flow cytometry. The
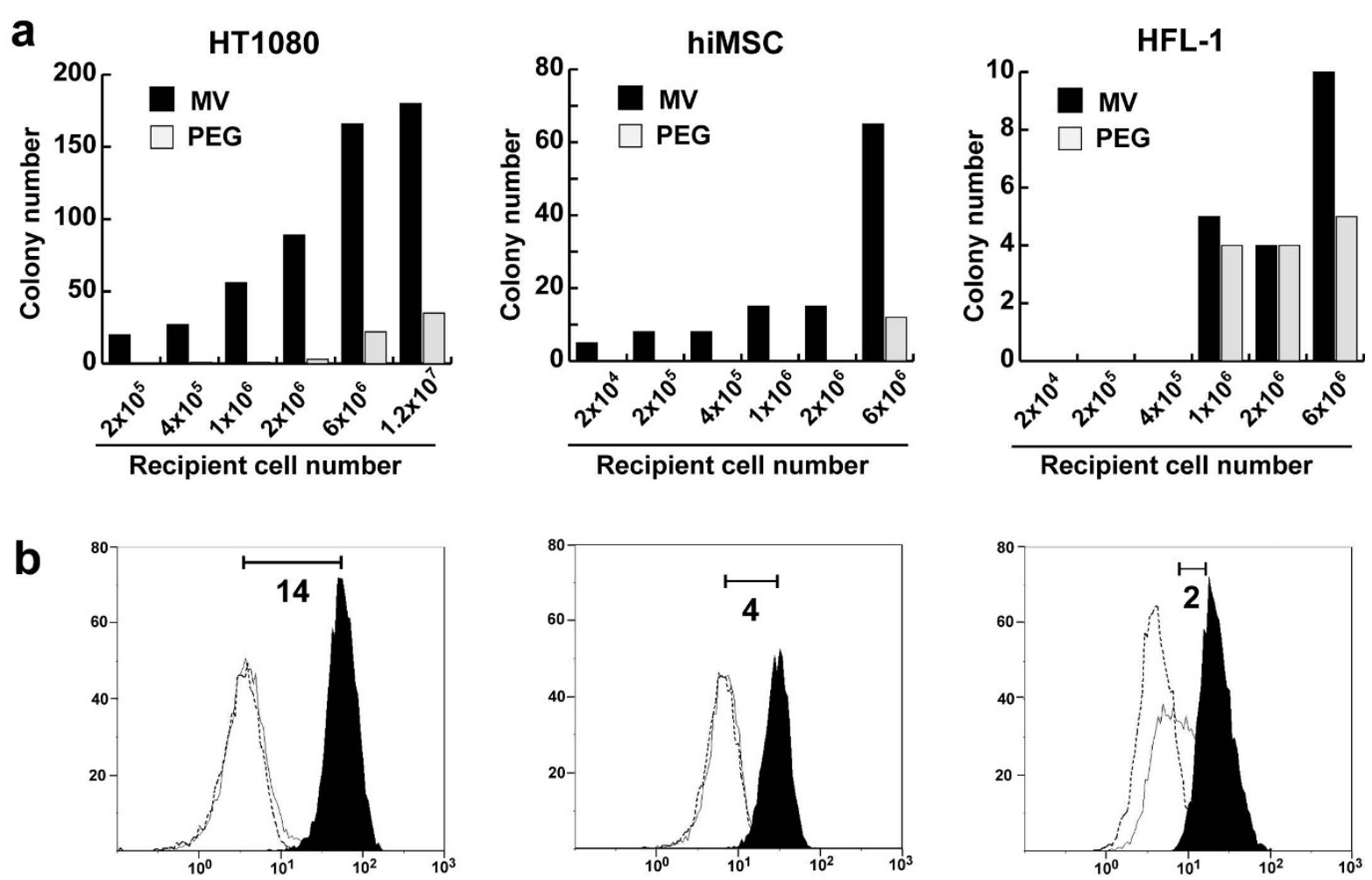

Figure 6 The efficiency of microcell fusion correlated with the surface density of CD46 in recipient cells. (a)A given number of microcells $(\sim 8$ $\times 10^{5}$ ) were applied to different numbers of recipient cells and fused using MV or PEG. Abundant microcell hybrids, assessed as the number of drugresistant colonies, were obtained using HT1080 and hiMSC cells. In contrast, few microcell hybrids were obtained using HFL-1 cells, irrespective of the fusogen used. (b) The expression level of CD46 on the cells was analyzed with flow cytometry by staining with FITC-conjugated anti-CD46 antibody (black peak) or an isotype control (white peak with solid line). No stained control was showed by white peak with dotted line. The numbers on the $x$ and y axes represent fluorescence intensity and count of event, respectively. CD46 expression is indicated by the number within the graph which is the ratio of the mean fluorescence index of the black:white peaks. 


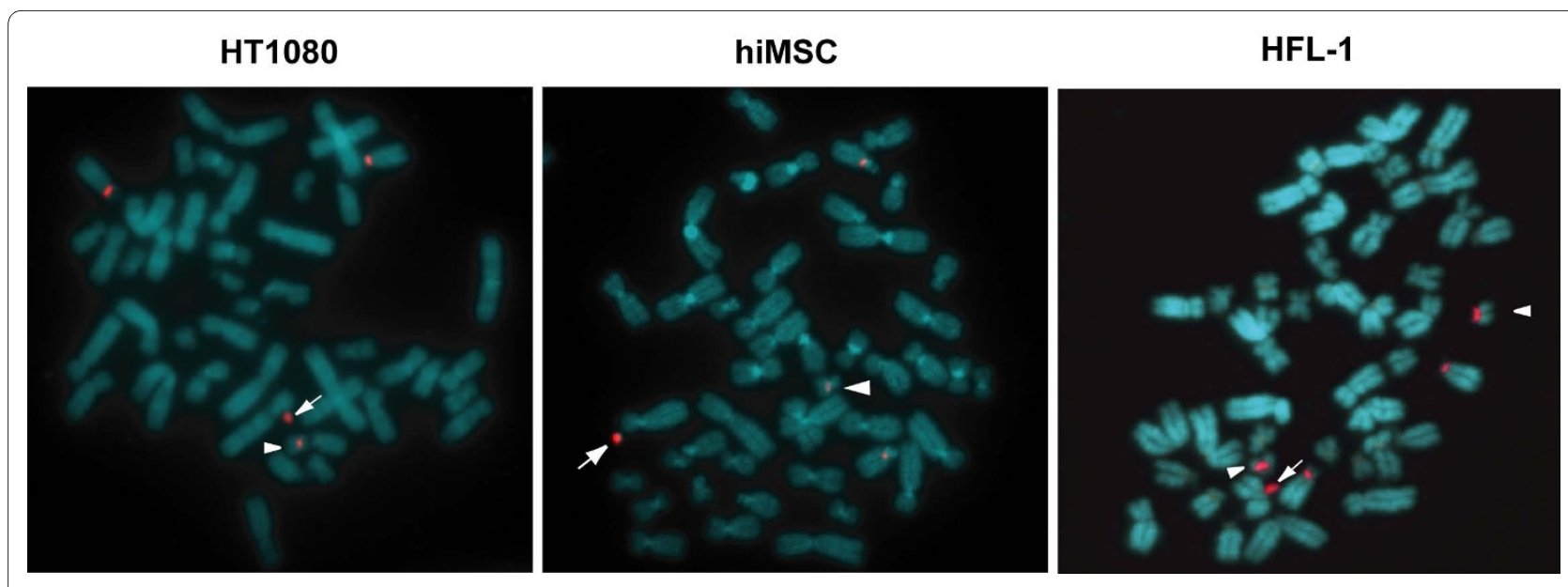

Figure 7 Detection of transferred HAC in microcell hybrids by FISH. The transferred HAC (arrow) is delineated by fluorescence in situ hybridization using an alphoid satellite probe derived from human chromosome 21. The alphoid satellite probe (red) detects endogenous chromosomes 13 and 21 (arrowheads), in addition to the HAC, due to their sequence similarity. Results from HT1080, hiMSC, or HFL-1 cells were placed on left, center, and right, respectively.

surface density of CD46 was quantified and expressed as the ratio of the mean fluorescence index of anti-CD46 antibody-stained and isotype control-stained cells. The HT1080, hiMSC, and HFL-1 cells showed high, moderate, and low levels of CD46 surface expression, respectively (Figure 6b). A correlation was noted between fusion efficiency and expression level of CD46. These results is consistent with the previous report of intercellular fusion in MV-infected human cells that cell fusion was minimal at low receptor densities but increased significantly above a threshold density of the receptor [28]. Therefore, measurement of the surface density of CD46 is a useful criterion for prediction of the ability of the MV fusogen to induce microcell fusion in recipient cells.

\section{Discussion}

We aimed to determine whether microcell fusion could be achieved using an MV fusogen. We showed that introduction of genes encoding MV envelope proteins enables rodent cells to produce fusogenic microcells that efficiently transmit donor chromosomes to recipient human cells expressing a high level of CD46 (Figure 1). This method has several advantages over the conventional PEG-fusion method for chromosome transfer. First, microcell hybrids can be obtained from a low number of recipients, even from recipients that are too low in number for hybrids to be obtained by PEG-fusion, as long as the recipients express the CD46 receptor over a threshold density. Second, the procedure for microcell fusion is extremely simple. Thus the formation of microcell hybrids requires only the addition of prepared microcells to recipient cells. This ease of application, which avoids the laborious tasks of handling a highly viscous PEG solution and performing repeated washout steps, may improve the reproducibility of microcell fusion. We obtained abundant microcell hybrids using MV-fusion even using donor cells that should have heterogeneity in copy number of the harbored MV-F/H expression plasmids. Further improvement in the fusion efficiency can be expected by clonal selection of donor cells with a high level of MV-F/H expression.

Application of microcells over a threshold amount inversely reduced the appearance of drug-resistant colonies. In principle, the collected microcells are composed of a heterogeneous population and the nature of the contained chromosome is not uniform. Hence, a microcell containing $\mathrm{HAC}$ is expected to yield drug-resistant colonies. In contrast, a microcell containing the $\mathrm{MV}-\mathrm{F} / \mathrm{H}-$ tagged donor chromosome can potentially induce secondary fusion between the microcell hybrid and the surrounding recipient cells, which ultimately reduces the survival of microcell hybrids carrying the HAC. Application of lower numbers of microcells may help to obviate the negative effects that occur due to carry-over of the genes encoding the MV-H/F proteins.

The next issue for microcell fusion via an MV fusogen is whether the tropism for recipient cells can be altered from the default CD46 receptor to arbitrary receptors. Engineering of viral tropism has been pursued for many gene therapy-based strategies [29-31]. An MV-Edmreverse genetics system has allowed the design and construction of recombinant MVs that are better suited for oncolytic applications. Retargeting of $\mathrm{MV}$ has been achieved by the addition of specificity domains to the extracellular (carboxyl) H-protein terminus. Thus, the addition of EGF or IGF1 to the H protein efficiently retargeted MV to CD46-negative rodent cells expressing the human EGF or IGF1 receptor, and caused extensive syn- 
cytium formation [32]. Furthermore, the addition of single chain antibody fragments to the $\mathrm{H}$ protein also efficiently retargeted MV to the EGF receptor, myeloma surface antigen CD38, or urokinase receptor [25,26,33]. These results suggest that chimeric $\mathrm{H}$ proteins containing an additional protein domain are functionally displayed on the surface of transduced cells. Thus these chimeric $\mathrm{H}$ proteins sustain the ability to interact with the targeted receptor, and can proceed to membrane fusion by cooperating with the F protein. Therefore, modification of the $\mathrm{H}$ protein potentially enables retargeting of fusogenic microcells to recipient cells of interest.

\section{Conclusions}

The MV-fusogen procedure for microcell fusion that has been demonstrated in this study, not only facilitates microcell fusion but also facilitates future manipulation of the cell targeting of microcells, by exploitation of previously established genetic engineering of the MV-H protein.

\section{Methods \\ Cell culture}

HT1080, and hiMSC cells were grown in Dulbecco's modified Eagle's medium (Sigma) supplemented with $10 \%$ fetal bovine serum (BioWest). HFL-1, obtained from the Riken cell bank, was grown in Ham's F12 medium (Sigma) supplemented with $15 \%$ fetal bovine serum. HAC donor $\mathrm{CHO}$ cells were grown in Ham's F-12 medium supplemented with $10 \%$ fetal bovine serum.

\section{MV envelope protein expression plasmids and transfection} Construction of the measles $\mathrm{H}$ protein expression plasmid pTNH6-H was previously described [20]. The plasmid pCAG-T7-F encodes the $F$ protein of the Edmonston-B strain under the control of CAG promoter (Nakamura, unpublished). The DsRed/neo expression plasmid, pDsRed-Monomer-N1, was purchased from Clontech. Plasmids were linearized by restriction digestion with PvuI (NEB) before transfection. HAC donor $\mathrm{CHO}$ cells $\left(8 \times 10^{4} /\right.$ well in 24 -well plate (Nunc))were cotransfected with $0.3 \mu \mathrm{g}$ each of pTNH6-H and pCAG-T7$\mathrm{F}$, and $0.25 \mu \mathrm{g}$ of pDsRed-Monomer-N1 using cationic lipid (Lipofectamine 2000; Invitrogen). At $24 \mathrm{~h}$ after transfection, the cells were re-plated at low density and selected for 14 days with $800 \mu \mathrm{g} / \mathrm{ml}$ of G418 (Nacalai). Drug-resistant cells were recovered as a mixed population.

HT1080 cells $\left(2 \times 10^{6} / 6 \mathrm{~cm}\right.$ dish $)$ were co-transfected with $0.4 \mu \mathrm{g}$ each of pTNH6-H and pCAG-T7-F using cationic lipid (Lipofectamine 2000). After culture for $6 \mathrm{~h}$, syncytium formation was tested under microscope.

\section{Co-culture assay}

Equal numbers $\left(1 \times 10^{5}\right)$ of G418-resistant $\mathrm{CHO}$ cells and HT1080 cells were plated in a 60-mm dish (Beckton Dickinson) and cultured for a period of 3 days and syncytium formation was determined microscopically.

\section{Microcell fusion}

The day before microcell fusion, recipient cells were trypsinized and counted. A given number of recipient cells $\left(2 \times 10^{4}, 2 \times 10^{5}, 4 \times 10^{5}, 1 \times 10^{6}, 2 \times 10^{6}\right.$, or $\left.6 \times 10^{6}\right)$ were plated in adequate culture vessel (96-well, 24-well, 12-well, 6-well, 60-mm diameter, and 100-mm diameter, respectively). Donor $\mathrm{CHO}$ cells, grown in T-25 flasks (Nunc), were treated with $0.1 \mu \mathrm{g} / \mathrm{ml}$ colcemid (Gibco) for $72 \mathrm{~h}$ to induce micronuclei formation. Flasks were filled with medium containing $10 \mu \mathrm{g} / \mathrm{ml}$ cytochalasin B (Sigma) and centrifuged for $1 \mathrm{~h}$ at $8,000 \mathrm{rpm}(11,899 \times \mathrm{g})$ in a JLA10.5 rotor (Beckman). Pellets containing a crude microcell preparation were recovered and passed through membranes of 8-, 5-, and 3- $\mu \mathrm{m}$ pore size (Whatman). Collected microcell preparations were used for fusion with recipient cells. For PEG fusion, microcells were suspended in DMEM medium (Sigma) containing $50 \mu \mathrm{g} / \mathrm{ml}$ PHA-P (Difco) and added to the recipient cells. After incubation at $37^{\circ} \mathrm{C}$ for $15 \mathrm{~min}$, the medium was discarded. The cells were then exposed to $50 \%(\mathrm{w} / \mathrm{v})$ PEG1500 (Roche) for $1 \mathrm{~min}$ and washed three times with serum free medium. On the day following PEG treatment, the cells were trypsinized, sparsely replated, and cultured for 14 days in medium with $3 \mu \mathrm{g} / \mathrm{ml}$ of Blasticidin (Funakoshi). For MV fusion, an aliquot of microcells prepared from $\mathrm{CHO} 4 \mathrm{H} 6.1 \mathrm{M}$ was overlaid on recipient cells and left for $24 \mathrm{~h}$. The cells were then trypsinized, sparsely replated, and cultured for 14 days in medium with $3 \mu \mathrm{g} / \mathrm{ml}$ of Blasticidin.

\section{FISH analysis}

Metaphase chromosomes were prepared from colcemidtreated cell cultures by hypotonic treatment with $0.075 \mathrm{M}$ $\mathrm{KCl}$ and methanol/acetate (3:1) fixation. Fluorescence in situ hybridization was carried out using the alphoid DNA probe p11-4 labeled with digoxigenin (Roche) [34]. The digoxigenin signal was detected with an anti-digoxigeninrhodamine complex (Roche). The chromosomes were counterstained with DAPI (Sigma). Photographs were taken using a CCD camera mounted on a fluorescence microscope (Nikon). Images were processed using the software attached to the microscope.

\section{Flow cytometry analysis}

Cells were dispersed by treatment with $0.2 \%$ EDTA/PBS, washed twice with PBS, and resuspended in ice-cold PBS containing $2 \%(\mathrm{w} / \mathrm{v}) \mathrm{BSA}$ at a concentration of $10^{6}$ cells/ 
$\mathrm{ml}$. The cells were then incubated for $60 \mathrm{~min}$ on ice with a 1:50 final dilution of FITC-labeled anti-CD46 antibody (clone E4.3; BD Pharmingen), or FITC-labeled isotype control (clone G155-178; BD Pharmingen). After washing with BSA/PBS, the cells were analyzed by flow cytometry using an EPICS ALTRA (Beckman Coulter).

\section{Additional material}

\section{Additional file 1 The Measles $\mathrm{H}$ protein is expressed on the surface of} the genetically engineered $\mathrm{CHO}$ cells. The surface expression of measles $\mathrm{H}$ protein on the $\mathrm{CHO} 4 \mathrm{H} 6.1 \mathrm{M}$ cells was analyzed by flowcytometry. The cells were stained with anti-measles $\mathrm{H}$ and AlexaFluor 647 secondary antibody (grey peak) or only secondary antibody (white peak with solid line). No stained control was showed by white peak with dotted line. Methods. Cells were dispersed by treatment with $0.2 \%$ EDTA/PBS, washed twice with PBS, and resuspended in ice-cold PBS containing 2\% (w/v) BSA at a concentration of $10^{6}$ cells $/ \mathrm{ml}$. The cells were then incubated for 60 min on ice with a 1:150 final dilution of the primary mouse monoclonal ascites antibody recognizing measles $\mathrm{H}$ protein (Clone CV1, CV4; Chemicon). Subsequently, the cells were washed with $2 \%$ (V/V) FBS/PBS and incubated for for an additional 30 min with 1:250 final dilution of Alexa Flour 647 conjugated goat anti-mouse lgG (Molecular Probes). After washing with BSA/PBS, the cells were analyzed by flow cytometry using MoFlo XDP (Beckman Coulter).

\section{Additional file $\mathbf{2}$ Microcell hybrids excluded the neo gene-tagged} donor chromosome. Microcell hybrids obtained by Blasticidin selection were assessed for sensitivity to G418. $10^{4}$ hybrid cells were plated in 12 well cluster and cultured for one week with or without antibiotics. Cells were fixed by methanol, stained with Giemza, and photographed. Microcell hybrids were sensitve to $\mathrm{G} 418$, indicating that the neo gene-tagged donor chromosome was eliminated from microcell hybrids.

\section{Authors' contributions}

MK designed the study, performed plasmid transfections and cell fusion assays, and drafted the manuscript. YK designed the study and helped to draft the manuscript. KK, NK and MT performed microcell fusions and FISH analyses. YN performed flow cytometry analyses. TN provided materials and contributed to the conception of the study. MO conceived of and supervised the study. All authors read and approved the final manuscript.

\section{Acknowledgements}

We thank Haruka Matsumori, Hidetoshi Hoshiya, Yoshinori Watanabe, Kei Hiramatsu for technical contributions, Hiromi Miyauchi for helpful assistance with the flow cytometry analyses, and Toshiaki Inoue for critical discussions. This study was supported in part by CREST Program grant from the Japan Science and Technology Agency (MO, YK) and PRESTO Program grant from Japan Science and Technology (TN).

\section{Author Details}

'Division of Human Genome Science, Department of Molecular and Cellular Biology, Faculty of Medicine, Tottori University, Yonago 683-8503, Japan, ${ }^{2}$ Chromosome Engineering Research Center, Tottori University, Yonago 683 8503, Japan, ${ }^{3}$ Department of Biomedical Science, Institute of Regenerative Medicine and Biofunction, Graduate School of Medical Science, Tottori University, Yonago 683-8503, Japan, 4 Division of Functional Genomics, Research Center for Bioscience and Technology, Tottori University, Yonago 6838503, Japan, ${ }^{5}$ Core Research for Evolutional Science and Technology (CREST) project, Japan Science and Technology Agency, Kawaguchi 332-0012, Japan, ${ }^{6}$ Core Facility for Therapeutic Vectors, The Institute of Medical Science, The University of Tokyo, Tokyo 108-8639, Japan and 7RNA and Biofunctions, Precursory Research for Embryonic Science and Technology (PRESTO) project, Japan Science and Technology Agency, Kawaguchi 332-0012, Japan

Received: 15 October 2009 Accepted: 6 May 2010 Published: 6 May 2010

\section{References}

1. Meaburn KJ, Parris CN, Bridger JM: The manipulation of chromosomes by mankind: the uses of microcell-mediated chromosome transfer. Chromosoma 2005, 114:263-274.

2. Oshimura M, Katoh M: Transfer of human artificial chromosome vectors into stem cells. Reprod Biomed Online 2008, 16:57-69.

3. Rutsch F, Gailus S, Miousse IR, Suormala T, Sagne C, Toliat MR, Nürnberg G, Wittkampf T, Buers I, Sharifi A, Stucki M, Becker C, Baumgartner M, Robenek H, Marquardt T, Höhne W, Gasnier B, Rosenblatt DS, Fowler B, Nürnberg $P$ : Identification of a putative lysosomal cobalamin exporter altered in the cblF defect of vitamin B-12 metabolism. Nat Genet 2009, 41:234-239.

4. Oshimura M, Barrett JC: Role of telomerase repressors. Eur J Cancer 1997, 33:710-715

5. Quaye L, Dafou D, Ramus SJ, Song HL, Maharaj AG, Notaridou M, Hogdall E, Kjaer SK, Christensen L, Hogdall C, et al.: Functional complementation studies identify candidate genes and common genetic variants associated with ovarian cancer survival. Hum Mol Genet 2009, 18:1869-1878

6. Haugen AC, Goel A, Yamada K, Marra G, Nguyen TP, Nagasaka T, Kanazawa S, Koike J, Kikuchi Y, Zhong X, Arita M, Shibuya K, Oshimura M, Hemmi H, Boland CR, Koi M: Genetic instability caused by loss of MutS homologue 3 in human colorectal cancer. Cancer Res 2008, 68:8465-72.

7. Mukaida N, Kodama S, Suzuki K, Oshimura M, Watanabe M: Transmission of genomic instability from a single irradiated human chromosome to the progeny of unirradiated cells. Radiat Res 2007, 167:675-681.

8. Nguyen P, Cui H, Bisht KS, Sun L, Patel K, Lee RS, Kugoh H, Oshimura M, Feinberg AP, Gius D: CTCFL/BORIS is a methylation-independent DNAbinding protein that preferentially binds to the paternal $\mathrm{H} 19$ differentially methylated region. Cancer Res 2008, 68:5546-5551.

9. Ishihara K, Oshimura M, Nakao M: CTCF-dependent chromatin insulator is linked to epigenetic remodeling. Mol Cell 2006, 23:733-742.

10. Tomizuka K, Yoshida H, Uejima H, Kugoh H, Sato K, Ohguma A, Hayasaka M, Hanaoka K, Oshimura M, Ishida I: Functional expression and germline transmission of a human chromosome fragment in chimaeric mice. Nat Genet 1997, 16:133-143.

11. Doherty $A M O$, Fisher EMC: Microcell-mediated chromosome transfer (MMCT): small cells with huge potential. Mamm Genome 2003, 14:583-592

12. Robl JM, Kasinathan $P$, Sullivan E, Kuroiwa $Y$, Tomizuka K, Ishida I: Artificial chromosome vectors and expression of complex proteins in transgenic animals. Theriogenology 2003, 59:107-113.

13. Shinohara T, Tomizuka K, Miyabara S, Takehara S, Kazuki Y, Inoue J, Katoh M, Nakane H, lino A, Ohguma A, Ikegami S, Inokuchi K, Ishida I, Reeves RH, Oshimura M: Mice containing a human chromosome 21 model behavioral impairment and cardiac anomalies of Down's syndrome. Hum Mol Genet 2001, 10:1163-1175.

14. Katoh M, Ayabe F, Norikane S, Okada T, Masumoto H, Horike S, Shirayoshi Y, Oshimura M: Construction of a novel human artificial chromosome vector for gene delivery. Biochem Biophys Res Commun 2004 321:280-290.

15. Hoshiya H, Kazuki Y, Abe S, Takiguchi M, Kajitani N, Watanabe Y, Yoshino T, Shirayoshi Y, Higaki K, Messina G, Cossu G, Oshimura M: A highly stable and nonintegrated human artificial chromosome (HAC) containing the 2.4 Mb entire human dystrophin gene. Mol Ther 2009, 17:309-317

16. Yang J, Shen MH: Polyethylene glycol-mediated cell fusion. Methods Mol Biol 2006, 325:59-66

17. Wojcieszyn JW, Schlegel RA, Lumley-Sapanski K, Jacobson KA: Studies on the mechanism of polyethylene glycol-mediated cell fusion using fluorescent membrane and cytoplasmic probes. J Cell Biol 1983, 96:151-9.

18. Golestani R, Pourfathollah AA, Moazzeni SM: Cephalin as an efficient fusogen in Hybridoma technology: Can it replace poly ethylene glycol? Hybridoma 2007, 26:296-301.

19. Roos DS, Choppin PW: Biochemical studies on cell fusion. I. Lipid composition of fusion-resistant cells. J Cell Biol 1985, 101:1578-90.

20. Roos DS, Choppin PW: Biochemical studies on cell fusion. II. Control of fusion response by lipid alteration. J Cell Biol 1985, 101:1591-8.

21. Grammatikos SI, Subbaiah PV, Victor TA, Miller WM: Diverse Effects of Essential (N-6 and N-3) Fatty-Acids on Cultured-Cells. Cytotechnology 1994, 15:31-50 
22. Navaratnarajah CK, Leonard VHJ, Cattaneo R: Measles Virus Glycoprotein Complex Assembly, Receptor Attachment, and Cell Entry. Curr Top Microbiol Immunol 2009, 329:59-76.

23. Blechacz B, Russell SJ: Measles virus as an oncolytic vector platform. Curr Gene Ther 2008, 8:162-175.

24. Grote D, Russell SJ, Cornu TI, Cattaneo R, Vile R, Poland GA, Fielding AK: Live attenuated measles virus induces regression of human lymphoma xenografts in immunodeficient mice. Blood 2001, 97:3746-3754.

25. Nakamura T, Peng KW, Vongpunsawad S, Harvey M, Mizuguchi H, Hayakawa T, Cattaneo R, Russell SJ: Antibody-targeted cell fusion. Nat Biotech 2004, 22:331-336.

26. Nakamura T, Peng KW, Harvey M, Greiner S, Lorimer IAJ, James CD, Russell SJ: Rescue and propagation of fully retargeted oncolytic measles viruses. Nat Biotech 2005, 23:209-214.

27. Okamoto T, Aoyama T, Nakayama T, Nakamata T, Hosaka T, Nishijo K, Nakamura T, Kiyono T, Toguchida J: Clonal heterogeneity in differentiation potential of immortalized human mesenchymal stem cells. Biochem and Biophys Res Commun 2002, 295:354-361.

28. Anderson BD, Nakamura T, Russell SJ, Peng KW: High CD46 receptor density determines preferential killing of tumor cells by oncolytic measles virus. Cancer Res 2004, 64:4919-4926.

29. Mathis JM, Stoff-Khalili MA, Curiel DT: Oncolytic adenoviruses - selective retargeting to tumor cells. Oncogene 2005, 24:7775-7791.

30. Jaras M, Brun ACM, Karlsson S, Fan XL: Adenoviral vectors for transient gene expression in human primitive hematopoietic cells: Applications and prospects. Exp Hematol 2007, 35:343-349.

31. Buchholz CJ, Muhlebach MD, Cichutek K: Lentiviral vectors with measles virus glycoproteins - dream team for gene transfer? Trends Biotechnol 2009, 27:259-265.

32. Schneider U, Bullough F, Vongpunsawad S, Russell SJ, Cattaneo R: Recombinant measles viruses efficiently entering cells through targeted receptors. J Virol 2000, 74:9928-9936.

33. Jing YQ, Tong CL, Zhang J, Nakamura T, lankov I, Russell SJ, Merchan JR: Tumor and Vascular Targeting of a Novel Oncolytic Measles Virus Retargeted against the Urokinase Receptor. Cancer Res 2009, 69:1459-1468.

34. Ikeno M, Masumoto H, Okazaki T: Distribution of CENP-B boxes reflected in CREST centromere antigenic sites on long-range alpha-satellite DNA arrays of human chromosome 21. Hum Mol Genet 1994, 3:1245-1257.

doi: $10.1186 / 1472-6750-10-37$

Cite this article as: Katoh et al., Exploitation of the interaction of measles virus fusogenic envelope proteins with the surface receptor CD46 on human cells for microcell-mediated chromosome transfer BMC Biotechnology 2010, 10:37

\section{Submit your next manuscript to BioMed Centra} and take full advantage of:

- Convenient online submission

- Thorough peer review

- No space constraints or color figure charges

- Immediate publication on acceptance

- Inclusion in PubMed, CAS, Scopus and Google Scholar

- Research which is freely available for redistribution

Submit your manuscript at www.biomedcentral.com/submit
C) Biomed Central 